\title{
COVID-19 with positive bronchoalveolar lavage fluid but negative nasopharyngeal and oropharyngeal swabs: A case report and insights
}

\author{
Reza Jahromi ${ }^{1}$, Arezoo Avazpour ${ }^{2}$, Maryam Jahromi ${ }^{3}$, Jalile Alavi ${ }^{4}$ \\ From ${ }^{1}$ Ph.D. Candidate, Department of Biomedical Engineering, Texas A\&M University, Texas, United States, ${ }^{2}$ Medical Intern, School of Medicine, \\ Azad University of Mashhad, Mashhad, Razavi Khorasan, Iran, ${ }^{3}$ Medical Doctor, Department of Family Physician, Jahrom University of Medical \\ Sciences, Jahrom, Iran, ${ }^{4}$ Medical Doctor, Department of Internal Medicine, Shiraz University of Medical Sciences, Shiraz, Fars, Iran
}

\begin{abstract}
Coronavirus disease 2019 (COVID-19) mainly affects the respiratory system, with some patients rapidly progressing to acute respiratory distress syndrome (ARDS). Nasopharyngeal and oropharyngeal swab specimens tested by real-time reverse transcription-polymerase chain reaction (RT-PCR) are the most commonly used methods to diagnose COVID-19. Herein, we investigate and discuss a 33-year-old case of COVID-19, without any preexisting medical conditions, whose both nasopharyngeal and oropharyngeal swabs were negative in the prodromal phase despite showing COVID-19 symptoms. However, after 3 days, with severe dyspnea and rapidly progressed ARDS, the patient was identified as infected by COVID-19 by testing bronchoalveolar lavage fluid (BALF). He was intubated in the intensive care unit (ICU) but expired on the $4^{\text {th }}$ day. This case shows the importance of active and accurate monitoring of the patients showing COVID-19 symptoms. If nasopharyngeal and oropharyngeal swabs are negative, a BALF test is recommended if performed by an expert operator.
\end{abstract}

Key words: Acute respiratory distress syndrome, Bronchoalveolar lavage fluid, COVID-19, SARS-CoV-2

I n December 2019, an outbreak of acute respiratory illness, named as the COVID-19 by the World Health Organization (WHO), emerged in Wuhan, China.[1] The disease quickly spreads to Chinese cities and other parts of the world. The novel coronavirus is caused by severe acute respiratory syndrome coronavirus-2 (SARS-CoV-2). The same as SARS-CoV, the novel coronavirus (SARS-CoV-2) uses angiotensin-converting enzyme 2 (ACE2) receptor to enter the host target cell but mainly spreads through the lower respiratory tract.[2] Previous reports show that fever (98\%), cough (76\%), dyspnea (55\%), myalgia, or fatigue $(44 \%)$ are the most common complaints of patients.[3-5] Gastrointestinal involvement, a(cute cardiac injury, and acute kidney injury caused by COVID-19 are also reported in other studies.[4,6]. By affecting the respiratory system, COVID19 rapidly progresses to acute respiratory distress syndrome (ARDS) in some patients and makes them likely to be admitted to the intensive care unit (ICU) or die.[7,8] Nasopharyngeal and oropharyngeal swab specimens tested by real-time reverse transcription-polymerase chain reaction (RT-PCR) are the most commonly used methods to diagnose COVID-19.

\section{Access this article online}

Received - 17 June 2020

Initial Review - 24 June 2020

Accepted - 06 July 2020

DOI: $10.32677 /$ IJCR.2020.v06.i07.010
In this report, we investigate and discuss an unusual case of COVID-19 who was not identified as infected in his first visit despite of performing the RT-PCR test using both nasopharyngeal and oropharyngeal swabs. However, the patient was again referred after 3 days, with a severely progressed ARDS, leading to his death.

\section{CASE REPORT}

A 33-year-old man was referred to the COVID-19 screening clinic of Abu-Ali-Sina Hospital (in Shiraz, Iran), with complaints of dry cough, sore throat, a fever of $38.2^{\circ} \mathrm{C}$, chills, muscle pain, and with no other apparent symptoms on April 28, 2020. He had no specific underlying disease, and he denied any involvement with people suspected of having COVID-19. His family members did not have COVID-19 either.

On arrival, he had a pulse rate of 80 beats/min, respiratory rate of 16 breaths $/ \mathrm{min}$, blood pressure of $115 / 75 \mathrm{mmHg}$, and oxygen saturation of $97 \%$ on room air, and he did not have dyspnea. Based on his symptoms, the patient was considered a suspected case of COVID-19, according to the Iran Ministry of Health Protocol for COVID-19. Therefore, an RT-PCR test by taking

Correspondence to: Reza Jahromi, Department of Biomedical Engineering, Texas A\&M University, College Station, TX 77843, USA. E-mail: reza.jahromi@tamu.edu

(C) 2020 The Author(s). This open access article is distributed under a Creative Commons Attribution (CC-BY) 4.0 license. 
both nasopharyngeal and oropharyngeal swab specimens was performed on him. The result was negative, and he was advised to stay home in quarantine to recover.

After 3 days of symptomatic treatment and taking antipyretic, including acetominophen $500 \mathrm{mg}$ ( 2 pills every $6 \mathrm{~h}$ ) and diphenhydramine citrate $(40 \mathrm{mg}$ orally every $6 \mathrm{~h}$ ), he did not improve, and on May 1, 2020, he was seen in the emergency department of this hospital with ARDS added to his previous symptoms. On admission, physical examination revealed vital signs as follows: A pulse rate of 140 beats/min, respiratory rate of 40 breaths $/ \mathrm{min}$, blood pressure of 100/60 $\mathrm{mmHg}$, and a fever of $38.5^{\circ} \mathrm{C}$. Lung auscultation revealed bilateral rhonchi, and arterial blood gas analysis indicated $\mathrm{pH} 7.26$, partial pressure of oxygen $\left(\mathrm{PaO}_{2}\right) 30 \mathrm{mmHg}, \mathrm{PaCO}_{2} 49 \mathrm{mmHg}, \mathrm{PaHCO}_{3} 21 \mathrm{mEq} / \mathrm{L}$, and oxygen saturation of $50 \%$ (on room air). The patient did not respond to non-mechanical support and had a short and rapid breath. Therefore, he was intubated, and mechanical ventilation was installed for him in the ICU. He was also prescribed propofol (50 $\mathrm{mg}$ intravenous infusion for $1 \mathrm{~h}$ ), midazolam (5 mg intravenous infusion for $1 \mathrm{~h}$ ), ketamine (50 $\mathrm{mg}$ intravenous infusion for $1 \mathrm{~h}$ ), hydrocortisone (200 mg stat), and furosemide (40 mg stat).

RT-PCR test was again performed on him, but this time, by taking a specimen from the bronchoalveolar lavage fluid (BALF), and the result was positive. A chest X-ray was also performed which revealed bilateral diffuse interstitial pulmonary edema with a left-sided pleural effusion (Figure 1). A complete blood count with the differential test was performed. Results showed leukopenia and neutropenia. However, the electrolyte level, myocardial enzymes, liver, and renal functions were all normal. Blood and sputum cultures were negative.

The antigen test for influenza $\mathrm{A}$ and $\mathrm{B}$ was negative. IgM test for influenza A and B, parainfluenza, respiratory syncytial virus, adenovirus, chlamydia pneumonia, mycoplasma pneumonia, and legionella pneumophila was negative. Blood and sputum cultures were performed before the administration of antibiotics, and results were negative. The patient's electrocardiogram showed sinus tachycardia, and there was no evidence of cardiac ischemia. Echocardiography was performed and demonstrated an ejection

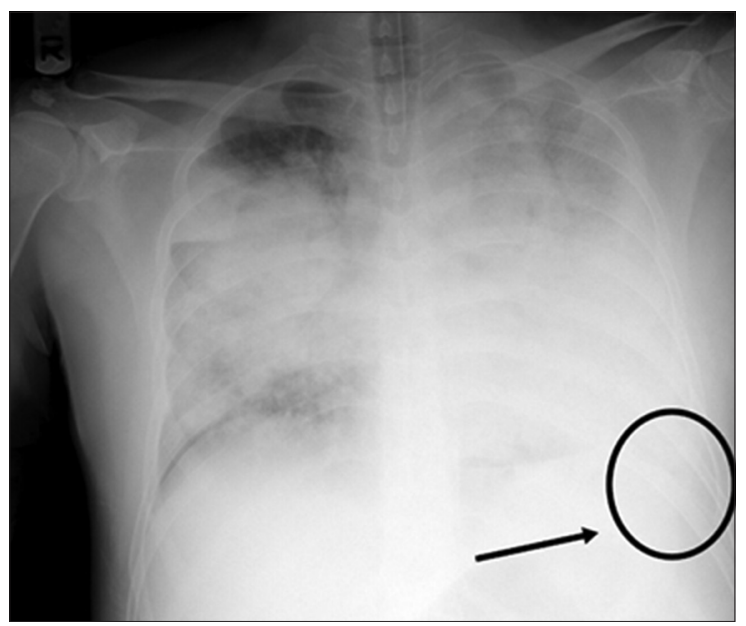

Figure 1: Chest X-ray of the patient on May 1, 2020, showing bilateral diffuse interstitial pulmonary edema with left-sided pleural effusion (as shown by arrow) fraction of $45 \%$, moderately dilated right ventricle (RV), and mild tricuspid regurgitation, with no clot in the pulmonary artery. On the $4^{\text {th }}$ day, symptoms of the patient did not improve, and he expired $28 \mathrm{~h}$ after admission.

The study was approved by the ethics committee of AbuAli-Sina Hospital on May 6, 2020 (No. 029802), and informed consent was obtained from the patient family for publication of this case report and accompanying image.

\section{DISCUSSION}

The WHO declared the outbreak of COVID-19 constitutes a Public Health Emergency of International Concern on January 30, 2020. The spectrum of this disease ranges from asymptomatic/ mild to severe/life threatening. $[9,10]$ SARS-CoV-2 has proven to be often deadly to the elderly and individuals with underlying disease or immunodeficiency,[11] while young patients with COVID-19 are less likely to progress to severe disease.[12]

The case of this study was neglected in the prodromal phase as he was young without any underlying disease and with negative nasopharyngeal and oropharyngeal RT-PCR tests. However, then, he was identified as infected by COVID-19 with severely progressed ARDS. This study shows that the general population is susceptible to SARS-CoV-2, and it is essential to give serious consideration to the youth or individuals without any preexisting medical conditions, and if any symptoms of COVID19 are observed, regardless of age, more accurate evaluation and follow-up should be performed.

The patient reported in this article was in a controversial position. The RT-PCR test of SARS-CoV-2 RNA using nasopharyngeal and oropharyngeal swabs was negative. In contrast, it was positive when a specimen was taken from the BALF. The reason is that the result of RT-PCR for identifying SARS-CoV-2 depends on the sample viral load. Angiotensinconverting enzyme-2 (ACE-2) is identified as the cell entry receptor of SARS-CoV-2. ACE-2 is expressed in the lungs more than the upper respiratory tract.[13] Therefore, depending on the site from which the specimen is taken, the RT-PCR test may result in false negative. This fact explains why the oropharyngeal or nasopharyngeal swab test is sometimes false negative, while the BALF specimen test might be positive. The case of this study had negative oropharyngeal or nasopharyngeal swabs but positive BALF. Therefore, a BALF test is recommended for the patients with this condition. Although the BALF test has a higher exposure risk, it is considered more accurate and recommended if performed by an expert operator.

In some COVID-19 patients, apart from the amount of virus load, the immune system of the body can cause rapid progression of lung injury, leading to respiratory failure. Cytokine storm is a severe immune response caused by COVID-19 infection. The occurrence of a cytokine storm can result in severe tissue damage. In this situation, as the lungs are damaged, efforts should be focused on suppressing the inflammation. Therefore, any anti-inflammatory agents can play an essential role in reducing 
the incidence of ARDS and, consequently, the mortality rate. A recent study [14] has also shown that in patients hospitalized with COVID-19, dexamethasone can reduce 28-day mortality among those receiving invasive mechanical ventilation. Therefore, we suggest that for the patients with rapidly progressed ARDS (such as the case of this study), the focus should not only be on antiviral medications but also more studies on immune system reactions should be performed.

\section{CONCLUSION}

The general population is susceptible to SARS-CoV-2. This study shows the necessity of active and accurate monitoring of individuals without any underlying disease or the youth who are showing symptoms of COVID-19. If the oropharyngeal or nasopharyngeal swabs of a patient are negative, while COVID19 symptoms are observed, a BALF specimen test should be considered. As the BALF test has a higher exposure risk, it is recommended to be performed by an expert operator.

\section{REFERENCES}

1. World Health Organization. Novel Coronavirus (2019-nCoV) Situation Report-22; 2020. Available from: https://www.who.int/emergencies/diseases/ novel-coronavirus-2019/situation-reports. [Last accessed on 2020 Jun 27].

2. Guo YR, Cao QD, Hong ZS, Tan YY, Chen SD, Jin HJ, et al. The origin, transmission and clinical therapies on coronavirus disease 2019 (COVID-19) outbreak-an update on the status. Mil Med Res 2020;7:11.

3. Song F, Shi N, Shan F, Zhang Z, Shen J, Lu H, et al. Emerging 2019 novel coronavirus (2019-nCoV) pneumonia. Radiology 2020;295:210-7.

4. Chen L, Liu H, Liu W, Liu J, Liu K, Shang J, et al. Analysis of clinical features of 29 patients with 2019 novel coronavirus pneumonia. Zhonghua Jie He He Hu Xi Za Zhi 2020;43:E005.

5. Jahromi R, Mogharab V, Jahromi H, Avazpour A. Synergistic Effects of
Anionic Surfactants on Coronavirus (SARS-CoV-2) Virucidal Efficiency of Sanitizing Fluids to Fight COVID-19. Cold Spring Harbor Laboratory: BioRxiv; 2020.

6. Wang D, Hu B, Hu C, Zhu F, Liu X, Zhang J, et al. Clinical characteristics of 138 hospitalized patients with 2019 novel coronavirus-infected pneumonia in Wuhan, China. JAMA 2020;323:1061-9.

7. Chan JF, Yuan S, Kok KH, To KK, Chu H, Yang J, et al. A familial cluster of pneumonia associated with the 2019 novel coronavirus indicating person-toperson transmission: A study of a family cluster. Lancet 2020;395:514-23.

8. Phan LT, Nguyen TV, Luong QC, Nguyen TV, Nguyen HT, Le HQ, et al. Importation and human-to-human transmission of a novel coronavirus in Vietnam. N Engl J Med 2020;382:872-4.

9. Grein J, Ohmagari N, Shin D, Diaz G, Asperges E, Castagna A, et al. Compassionate use of remdesivir for patients with severe COVID-19. N Engl J Med 2020;382:2327-36.

10. Hu Z, Song C, Xu C, Jin G, Chen Y, Xu X, et al. Clinical characteristics of 24 asymptomatic infections with COVID-19 screened among close contacts in Nanjing, China. Sci China Life Sci 2020;63:706-11.

11. Nikolich-Zugich J, Knox KS, Rios CT, Natt B, Bhattacharya D, et al. SARS-CoV-2 and COVID-19 in older adults: What we may expect regarding pathogenesis, immune responses, and outcomes. Geroscience 2020;42:505-14.

12. Liu K, Chen Y, Lin R, Han K. Clinical features of COVID-19 in elderly patients: A comparison with young and middle-aged patients. J Infect 2020;80:e14-8.

13. Lu R, Zhao X, Li J, Niu P, Yang B, Wu H, et al. Genomic characterisation and epidemiology of 2019 novel coronavirus: Implications for virus origins and receptor binding. Lancet 2020;395:565-74.

14. Horby P, Lim WS, Emberson J, Mafham M, Bell J, Linsell L, et al. Effect of Dexamethasone in Hospitalized Patients with COVID-19: Preliminary Report. Cold Spring Harbor Laboratory: MedRxiv; 2020.

Funding: None; Conflicts of Interest: None Stated.

How to cite this article: Jahromi R, Avazpour A, Jahromi M, Alavi J. COVID-19 with positive bronchoalveolar lavage fluid but negative nasopharyngeal and oropharyngeal swabs: A case report and insights. Indian J Case Reports. 2020;6(7):380-382. 